\title{
ENHANCING INTEROPERABILITY THROUGH THE ONTOLOGICAL FILTERING SYSTEM
}

\author{
Raffaello Lepratti, Ulrich Berger \\ Brandenburg University of Technology at Cottbus, Chair of Automation Technology \\ D-03013 Cottbus, GERMANY \{lepratti,ulrich-berger\}@aut.tu-cottbus.de
}

\begin{abstract}
European industry is in transition process from a mass production industry towards a knowledge-based customer- and service-oriented one. Consequently, networked, knowledge-driven and agile manufacturing systems emerge as key elements towards the future production scenario. In order to automate production tasks also for small production batch and enable uncomplicated system reconfigurations, a holistic knowledge management architecture, which enables knowledge acquisition and retrieval, is needed. Since several heterogeneous technologies and human actors are still involved in the knowledge transfer process interoperability barriers must be overcome. In this paper an ontology-based approach will be described. It enhances HumanMachine Interoperability simplifying the interaction level between them and makes possible automatic knowledge transfer. First trial tests have been performed by means of a natural language interaction system within the automation technology domain.
\end{abstract}

\section{THE EUROPEAN MANUFACTURING PARADIGM}

European industry is in transition process from a mass production industry towards a knowledge-based customer- and service-oriented one, which provides production on demand, mass customization, rapid reaction to market changes and quick time-tomarket of new innovative products.

In this transition, it faces the challenge to produce according to a lot-size one paradigm at low cost and high quality. Customizing in product development and consequently high variety of product features lead to a strong individualisation in the production domain. This influences the normal course of the product life cycle making it unforeseeable and risky concerning with the enterprise investment and resource plans (see Figure 1).

Following this vision, networked, knowledge-driven and agile manufacturing systems emerge as necessary key elements towards this future production scenario, which shall allow to European industry long-term competitiveness improvements above all by added values in product-services. 
Therefore, research efforts must focus on the development of new strategies providing an holistic solution for a knowledge-based manufacturing architecture. Its structure must feature a systemic dynamic learning behavior, in which innovation arises from new complex interaction forms between integrated technologies, human resources, management and organizations in all phases of the valued-added chain, i. e. (i) production preparation, (ii) planning and programming as well as (iii) process execution. New solutions shall aim at the development of a global concept, where new operational knowledge-acquisition and knowledge-feedback mechanisms as well as human machine interaction enable machine intelligence are embedded, in order to reach the required, challenging level of efficiency and robustness.

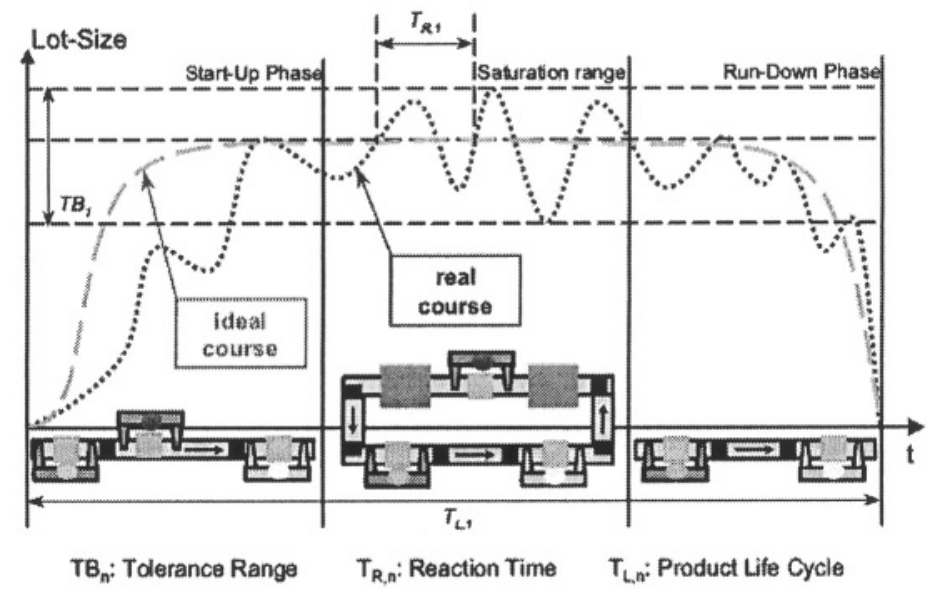

Figure 1: Customizing effect on the product life cycle.

Both, already existing and arising industrial know-how should be gathered together either manually from human experiences (usually by experts) and by use of intelligent cognitive sensing systems, or automatically deriving from human interventions (i. e. short process corrections at shop-floor level) and, if necessary, also retrieved. Knowledge-filtering and dispatching procedures provide standardization of knowledge form and task-oriented as well as user-based redistribution of the stored information. Such a novel workflow architecture is shown in Figure 2.

\section{INTEROPERABILITY ISSUES}

If, on the one hand, the workflow architecture proposed in Figure 2 could represent an adequate solution to meet the growing market challenges, on the other hand, it shows to be also ambitious in connection with its requirements.

A smooth global information flow between all actors involved in this process is the most important pre-requirement for ensuring the correct process behavior. However, still too many complications evolve when trying to find standard criteria for interoperability across the entire heterogeneous human qualifications and machine programming languages setting. 
As already stressed in (Lepratti and Berger, 2003), interoperation barriers could be identified in each of the main communication forms, which characterized today's enterprise environment: beginning from Human-Human and Machine-Machine communication till to Human-Machine interaction.

On the one hand, possible understanding problems i. e. arise, while two persons try to communicate with each other as consequences of discrepancies in their cultural and/or professional backgrounds. They are incline to cognitive perceive and mentally process same situations of the real world in different ways, referring these to different models (so called mental models). Thus, also two interaction partners, even though speaking the same language and using identical terminologies, can misunderstand each other, since vocabulary terms represent merely etiquettes of cognitive categories.

On the other hand, incompatibilities are the major reason of impediments in transferring data from a software system to another one, which uses distinct technology solutions (i. e. code languages), since applied syntax and semantic rules are structured in different ways.

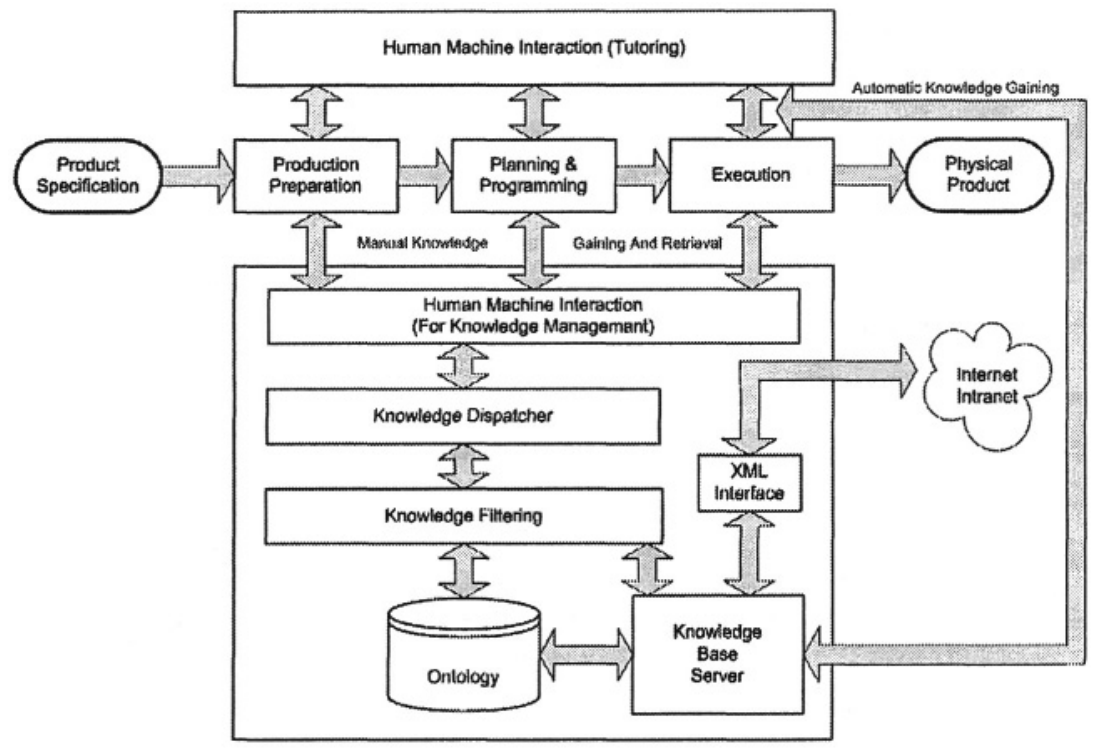

Figure 2: Building blocks solution for a knowledge-based architecture.

\section{STATE-OF-THE-ART}

Some standards enabling interoperability between manufacturing agents have been already successfully employed. There are three first relevant examples: The KIF (Knowledge Interchange Format) (Genesereth and Fikes, 1992), which allows interchange of knowledge among disparate software with the possibility to structurally represent knowledge at meta- level. The STEP ISO 10303 (STandard for the Exchange of Product data) (Fowler, 1995), which addresses to the exchange and sharing of information required for a product during its life cycle (such as 
parametric data like design rationale, functional specification and design intent). STEP is nowadays a well-known standard for real world product information modelling, communication and interpretation. Finally, The CORBA (Common Object Request Broker Architecture) (Object Management Group, 1995), which provides neutral - both platforms and languages independent-communication between remote applications based on object oriented distributed technology, allowing different clients and/or servers connected within a network to live as individual entities able to access to the information they need in a seamless and transparent way.

While the shown approaches are successfully proved and employed, they are to strong task-oriented and remain just one-off solutions. At present, a generic knowledge management concept for an architecture, as pictured in Figure 2, hasn't been developed until now. Too many resources are still used on knowledge retrieval. These resources are always difficult to plan and the extent of knowledge transfer between different projects and applications is often strong limited. The lack of generic architectures gives, therefore, present integrated systems a proprietary character, where capability in upgrading different heterogeneous systems, transparent data exchange among them, distributed open environments and improved information sharing represent the most important requirements.

\section{THE ONTOLOGICAL APPROACH}

The ontology as formal, explicit specification of a shared conceptualisation (Gruber, 1993) represents a well-known issue. Various relevant projects such as for example Ontosaurus (Swartout, 1996), Ontolingua (Gruber, 1993), Ontobroker (Decker, 1999), OntoSeek (Guarino et al., 1999), OntoWeb (Dörr et. al., 2001) and so on, confirm a current increasing interest in employing ontology-based approaches as emerging technology for enabling semantic independence of information providing a set of well defined rules, with which ambiguities in the communication between distinct software and human agents are avoided. Although the use of ontologies covers several domains such as knowledge management, knowledge bases, natural language processing, information access and agents, a lack in the literature is to observe concerning approaches in Human-Machine interaction. As logical consequence of a drastic growing automation degree (World Robotics, 2003), the relevance of an improved Human-Machine interoperation is stressed as one of the essential key issues for the future production scenario.

Thus, referring to Figure 2, also the design and implementation of mechanisms for easy Human-Machine interactions is of a paramount importance for the successful working of the entire architecture. The existing interaction forms and machine programming languages are still implemented in heterogeneous ways and bar the possibility of embedding human skills.

At shop-floor level, no available online methodology can handle significant exchange of HW/SW components. For instance manufacturing systems must be stopped and reprogrammed every time, when a reconfiguration is required.

In the next section it will be shown how an ontology approach based on the syntactic and semantic descriptions of natural languages - the so called Ontological Filtering System (OFS) - can meet the missing issue mentioned above, leading to a 
double advantage: Improvement of the Human-Machine interaction as well as acquirement and transfer of knowledge arising from human interventions.

\section{THE ONTOLOGICAL FILTERING SYSTEM}

According to the methodology discussed in (Lepratti and Berger, 2004) an ontology has been created for a variety of purposes within the automation technology domain. As case of study the PLC-based didactic system of Figure 3 was considered.

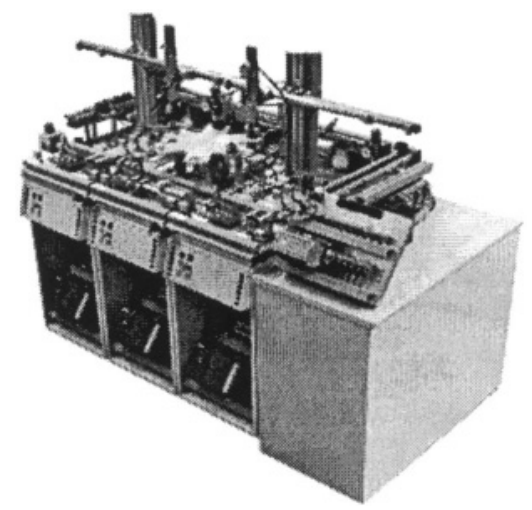

Figure 3: PLC System: OFS case of study.

The development foresees the following main steps:

a) Fixing Basis Terminology $\boldsymbol{W}$ consisting in a set of basic terms $\boldsymbol{W}_{\boldsymbol{N}}$, which have to be fixed by recognizing principal elements playing a significant role within the considered process as well as a set of possible actions $\boldsymbol{W}_{\boldsymbol{\nu}}$ among them. Some examples in case of the system of Figure 3 are listed below:

$W_{N}=\left\{w_{N 1}, w_{N 2}, . ., w_{N n}\right\}=\{$ conveyor, gripper arm, ramp, turntable $\ldots\}$

$W_{V}=\left\{w_{V l}, w_{V 2}, \ldots, w_{V n}\right\}=\{g r a s p$, open, move ... $\} W=W_{N} u W_{V}$

b) Terminology Enrichment Due to disparate possibilities of the natural language expression, a major number of terms, that could be used for the same intended meaning, have to be found out and included in $\boldsymbol{W}_{\boldsymbol{N}}$ and $\boldsymbol{W}_{\boldsymbol{V}}$ For this purpose a survey must be carried out trying to interview the largest number of persons with different professional backgrounds and/or different nationalities. Since for instance the English language bases on a lexicon of more than two hundred thousands different terms the collected terminology can be further enriched for instance using the WordNet $^{\odot}$ electronic lexical database (Fellbaum, 1998).

c) Chaining Up Terms Terms - either Noun or Verbs - could refer to the same meaning but with different degree of granularity in their level of specification. So, e. g. there are terms, which are general in their expression and others that go very deep with their meaning. The first ones could be used for everybody in different 
circumstances without owing special knowledge in the domain of use (in this case called Everyone Terminology), while the second ones are normally employed only by experts (in this case called Expert Terminology). All terms, which can be adopted within the same knowledge domain, are chained up, in order to form so called Synsets. Terms belonging to the same Synonymy-Set have following features:

- They refer to the same basis term within the knowledge domain of application

- They are connected to each other per different semantic relations (synonym, antonym, hyponym and so on)

- They have different granularity in their semantic level of specification

- They have a shared meaning for users.Figure 4 shows an example of Synset for the basic term gripper arm. On its top extremity Entity represents the correlated term with the most general meaning, while gripper arm is placed on the bottom as it shows the most specific meaning.

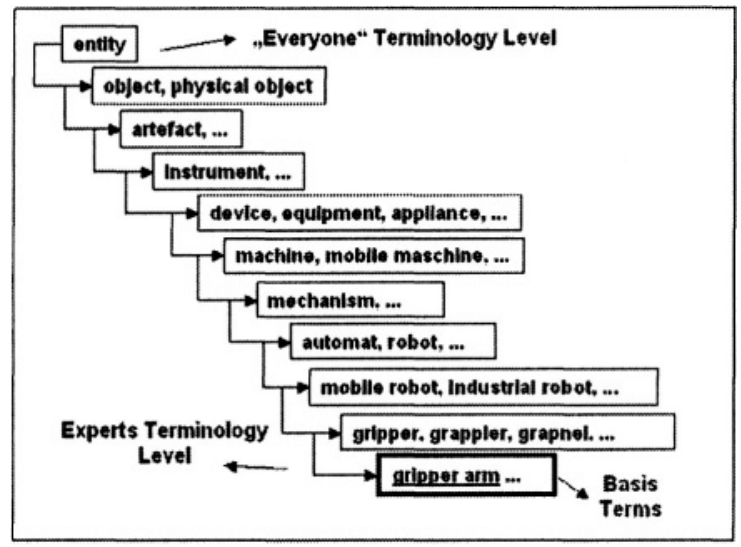

Figure 4: A sample of Synset for the basis term gripper arm.

d) Structuring Ontology Network This step concerns in merging all Synsets of all fixed base terms defined at point "c" into a whole semantic network called Ontological Network, This occurs in four steps: (i) identifying identical terms belonging to different Synsets, (ii) aligning these, (iii) merging corresponding Synsets into each other and (iv) building new semantic relations and/or recognizing and removing semantic inconsistencies.

e) Finally Semantic Classes such as Humans, Machines, Processes, Failures, Work Tools, Work Pieces as well as Relations among them have been defined, in order to gather terms together in one group according to their acquired semantic role in the domain of application and constrain their possible semantic combinations.

\section{TESTS AND CONCLUSION}

On the base of some pictures representing the considered objects, hundreds of persons working in the distinct professional sectors (e. g. pharmacists, bakers, students of different faculties, artists, philosophers, teachers an so on) and in the most cases also specking different languages (in particular English, German, Italian 
and Spanish) have been interviewed. An extract of survey results is illustrated in Table 1. The chosen describing language is English.

An extract of at present 500 collected terms by the performed survey of Table 1 is given on the basis of three base terms examples: conveyor, grasper and ramp.

According to these results, terms have been chained up in hierarchies according to their semantic specification degree (granularity). Terms belonging to more then one hierarchy have been localized (see Table 1) and used as connection points for merging the hierarchies into the whole ontological network as described in section 5 .

In order to test the obtained results an experimental environment has been set up. This consists of a natural language interaction system, which includes the Ontological Filtering System (OFS) (see Figure 5.). Its structure foresees also a semiotic, a syntax and a semantic analysis step as well as a machining coding and model checking level. It allows at present filtering and standardization of natural expression forms and, at the same time, translates entered, syntactically and semantically plausible instructions in machine codes.

First trial tests within the OFS have been performed. These showed interesting results, motivating further research activities in this field. Additional tests within a heterogeneous machine environment (RC, PLC and NC) are foreseen. These should prove concept suitability towards a future use in production context.

\begin{tabular}{|l|l|l|l|}
\hline Level & \multicolumn{1}{|c|}{ Synset 1 } & \multicolumn{1}{c|}{ Synset 2 } & \multicolumn{1}{|c|}{ Synset 3 } \\
\hline Basis Term & \multicolumn{1}{|c|}{ conveyor } & \multicolumn{1}{|c|}{ gripper arm } & \\
\hline & & & \\
& & & \\
& & & \\
& & & \\
\hline Term 1 & conveyer & grip & \\
\hline Term 2 & band & gripper & chute \\
\hline Term 3 & transporter & holder & transporter \\
\hline Term 4 & rail & clasp & panel \\
\hline Term 5 & drive & mounting & descent \\
\hline Term 6 & mechanism & robot & device \\
\hline Term 7 & device & fixer & rail \\
\hline Term 8 & assembly line & tool & track \\
\hline Term 9 & treadmill & device & ramp \\
\hline Term 10 & rail & grappler & entity \\
\hline Term 11 & construction & implement & fixed device \\
\hline Term 12 & instrument & holding device & entity \\
\hline Term 13 & entity & instrument & artefact \\
\hline Term 14 & moveable device & constraint & object \\
\hline$\ldots .$. & $\ldots .$. & $\ldots .$. & $\ldots .$. \\
\hline & & & slide \\
\hline
\end{tabular}

Table 1: An extract of performed survey 


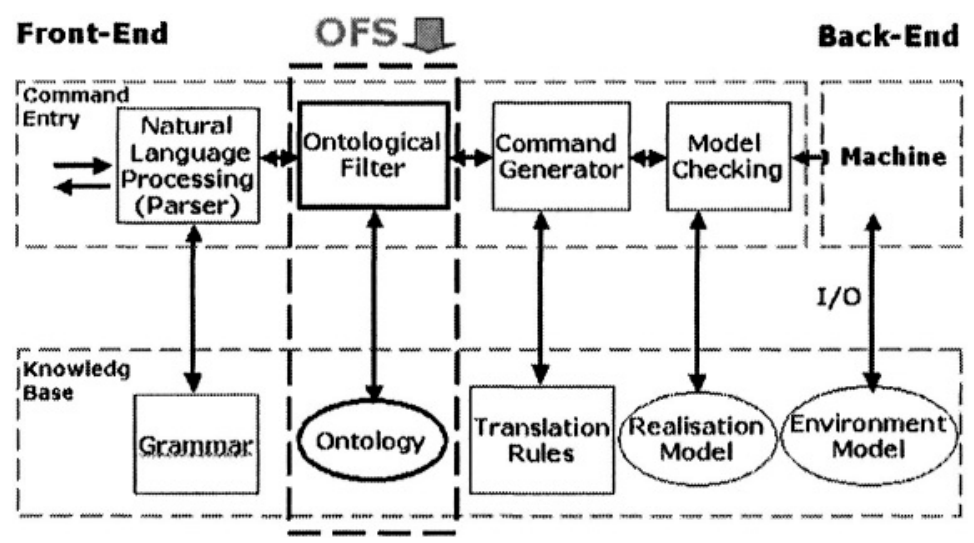

Figure 5: Structure of the natural language interaction system.

\section{6: ACKNOWLEDGMENT}

The project described in this paper was performed in the laboratory of the Department of Automation Technology of the Brandenburg University of Technology at Cottbus. The described trial tests have been carried out with a FESTO-Didactic System.

\section{REFERENCES}

1. Gruber, T. R. (1993): “A Translation Approach to Portable Ontology Specification”. In: Knowledge Acquisition 5, 1993, pp. 199-220.

3. Dörr, M.; Guarino N.; Fernández López, M; Schulten, E.; Stefanova, M.; Tate, A.: "OntoWeb: state of the Art in Content Standards". Report of the EU research project IST-2000-29243, November, 2001.

4. Lepratti R., Berger, U. (2003): "Towards Ontology Solutions for Enabling Interoperability in Virtual Enterprises". In: Processes and Foundations for Virtual Organisations (L. M. Camarinha-Matos and H. Afsarmanesh (Ed.)). Proceedings of the $4^{\text {th }}$ IFIP Working Conference in Virtual Enterprises (PRO-VE 2003), Kluwert, Boston-London , pp. 307-314.

6. Genesereth, M. R. and Fikes, R. E. (1992): Knowledge interchange format, version 3.0, reference manual. Technical report, Logic-92-1, Computer Science Dept., Stanford University, 1992.

7. Fowler, J. (1995): "STEP for Data Management, Exchange and Sharing", Technology Appraisals.

8. Object Management Group (1995): "The Common Object Request Broker Architecture and Specification (CORBA)", Revision 2.0, July, 1995

9. World Robotics (2003): "Statistics, Market Analysis, Forecasts, Case Studies and Profitability of Robot Investment", USA; 2003.

10 Lepratti, R.; Berger, U. (2004): "Improving e-Manufacturing Efficiency through the Ontological Filtering System". In: Proceedings of the 11th IFAC Symposium on Information Control Problems in Manufacturing, Salvador da Bahia, Brasil, April 5-7, 2004.

11. Swartout, B.; Patil, R., Knight, K.; Russ, T. (1996): "Toward Distributed Use of Large-Scale Ontologies”. In: Proceedings of KAW'96, November, Banff, Canada, 1996.

12. Decker, S; Erdmann, M.; Fensel, D.; Studer, R. (1999): "Ontobroker: Ontology Based Access to Distributed and Semi-Structured Information". In: Semantic Issues in Multimedia Systems, R. Meersman et. al. (eds.), Proceeding of DS-8, Kluwer Academic Publisher, Boston, pp. 351-369.

13. Guarino, N.; Masolo, C.; Vetere, G.: "OntoSeek: Content-Based Acess to the Web", in IEEE Intelligent System and Their Application. 14(3), 1999, pp. 70-80.

14. Fellbaum, C.: "WordNet: An Elektronik Lexical Database", MIT Press, USA, 1998. 